\title{
maxRatio improves the detection of samples with abnormal amplification profiles on QIAgen's artus HIV-1 qPCR assay
}

\section{[version 1; peer review: 1 approved]}

\author{
Luigi Marongiu(D1, Eric B. Shain², Marianna Martinelli3, Matteo Pagliari4, \\ Heike Allgayer ${ }^{1}$ \\ ${ }^{1}$ Department of Experimental Surgery, University of Heidelberg, Medical Faculty in Mannheim, Mannheim, Baden-Wuttenberg, \\ 68167, Germany \\ ${ }^{2}$ Grove Street Technology LLC, Grove Street Technology LLC, Glencoe, Illinois, 60022, USA \\ ${ }^{3}$ Department of Medicine and Surgery, University of Milano Bicocca, Monza, Italy, 20900, Italy \\ ${ }^{4}$ Department of Diagnostics in Animal Health, Istituto Zooprofilattico Sperimentale delle Venezie, Legnaro, Veneto, 35020, Italy
}

V1 First published: 24 Aug 2020, 9:1030

https://doi.org/10.12688/f1000research.25738.1

Second version: 29 Jun 2021, 9:1030

https://doi.org/10.12688/f1000research.25738.2

Latest published: 31 Aug 2021, 9:1030

https://doi.org/10.12688/f1000research.25738.3

\section{Abstract}

Background: Accurate viral load (VL) determination is paramount to determine the efficacy of anti-HIV-1 therapy. The conventional method used, fit-point (FP), assumes an equal efficiency in the polymerase chain reaction (PCR) among samples that might not hold for low-input templates. An alternative approach, maxRatio, was introduced to compensate for inhibition in PCR.

Methods: Herein, we assessed whether maxRatio could improve VL quantification using 2,544 QIAgen artus HI virus-1 RT-PCR reactions. The assay's standard dilutions were used to build external standard curves with either FP or maxRatio that re-calculated the VLS.

Results: FP and maxRatio were highly comparable (Pearson's $\rho=0.994$, Cohen's $\mathrm{k}=0.885)$, and the combination of the two methods identified samples $(n=41)$ with aberrant amplification profiles.

Conclusions: The combination of maxRatio and FP could improve the predictive value of the assay.

Keywords

HIV, qPCR, quantification, maxRatio, viral load.

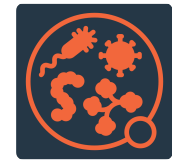

This article is included in the Pathogens

gateway.

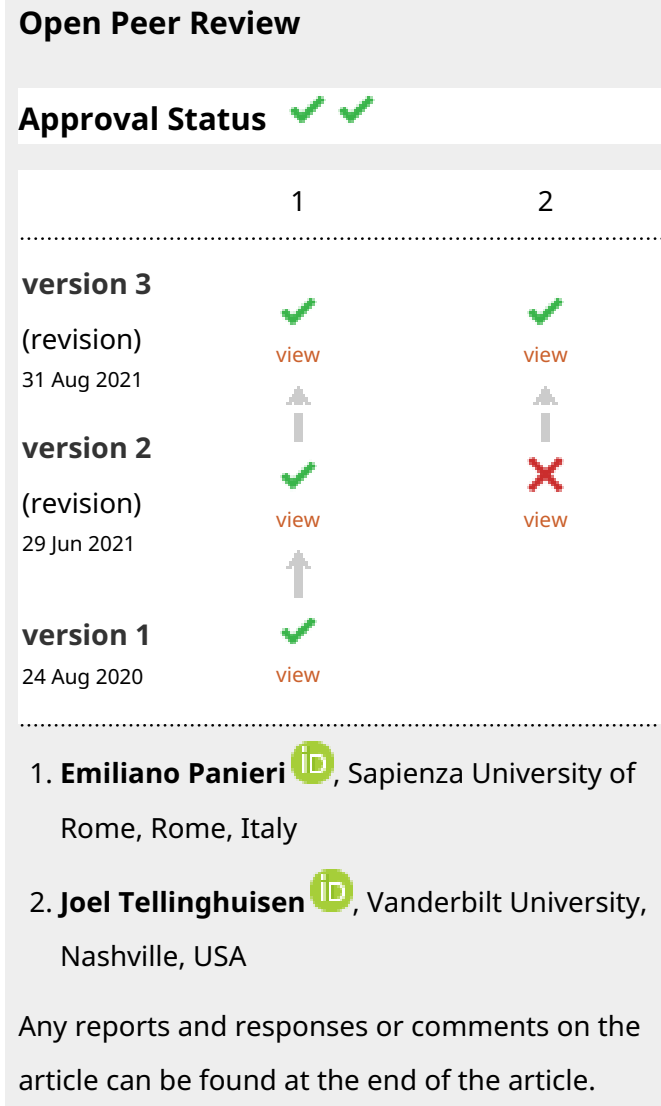


Corresponding author: Luigi Marongiu (luigi.marongiu@outlook.com)

Author roles: Marongiu L: Conceptualization, Data Curation, Formal Analysis, Investigation, Methodology, Supervision, Validation, Visualization, Writing - Original Draft Preparation, Writing - Review \& Editing; Shain EB: Conceptualization, Formal Analysis, Software, Validation; Martinelli M: Formal Analysis, Validation; Pagliari M: Formal Analysis, Validation; Allgayer H: Funding Acquisition, Project Administration, Supervision

Competing interests: No competing interests were disclosed.

Grant information: This work was supported by the Alfried Krupp von Bohlen und Halbach Foundation, Essen; the Deutsche Krebshilfe, Bonn (70112168); the Deutsche Forschungsgemeinschaft (DFG, grant number AL 465/9-1); the HEiKA Initiative (Karlsruhe Institute of Technology/University of Heidelberg collaborative effort); Dr Hella-Buehler-Foundation, Heidelberg; Ingrid zu Solms Foundation, Frankfurt; the DKFZ-MOST Cooperation, Heidelberg (grant number CA149); the HIPO/POP-Initiative for Personalized Oncology, Heidelberg (H032 and H027).

The funders had no role in study design, data collection and analysis, decision to publish, or preparation of the manuscript.

Copyright: $\odot 2020$ Marongiu L et al. This is an open access article distributed under the terms of the Creative Commons Attribution License, which permits unrestricted use, distribution, and reproduction in any medium, provided the original work is properly cited.

How to cite this article: Marongiu L, Shain EB, Martinelli M et al. maxRatio improves the detection of samples with abnormal amplification profiles on QIAgen's artus HIV-1 qPCR assay [version 1; peer review: 1 approved] F1000Research 2020, 9:1030 https://doi.org/10.12688/f1000research.25738.1

First published: 24 Aug 2020, 9:1030 https://doi.org/10.12688/f1000research.25738.1 


\section{Introduction}

Infection with HIV-1 accounts for a global prevalence of 38 million cases and a one million deaths yearly ${ }^{1}$. An accurate viral load (VL), typically carried out by quantitative polymerase chain reaction ( $\mathrm{qPCR}$ ), is pivotal for addressing the efficacy of antiviral therapies ${ }^{2}$. The threshold level of detection for the HIV-1 (VL) has been reported in the range 20-44 viral genomic copies per milliliter $(\mathrm{c} / \mathrm{mL})^{3,4}$.

qPCR data are usually analyzed by the fit-point (FP) method, which assumes equal amplification efficiency between samples ${ }^{5}$. However, anomalies in the background fluorescence at low template input, can affect the quantification ${ }^{6-8}$. An alternative method, maxRatio, was introduced to overcome these issues ${ }^{9}$. It has been reported that maxRatio conferred a marginal increase in assay accuracy over $\mathrm{FP}^{10,11}$.

FP provides only a quantification cycle $(\mathrm{Cq})$ value, which is then used to calculate VL. MaxRatio, instead, gives two parameters: one associated with the reaction's efficiency (MR) and one equivalent to, albeit distinct from, Cq. These two parameters can be linked to bestow a quantitative cycle (FCNA) compensated for inhibition.

In the present work, we aimed to determine whether maxRatio could improve the determination of HIV-1 VL. We compared the quantification of HIV-1 VL computed by FP and maxRatio on a dataset generated with the QIAgen artus HIV assay, which has a reported limit of detection of $35.5 \mathrm{c} / \mathrm{mL}$, and we showed that maxRatio could pinpoint samples with abnormal amplification profiles.

\section{Methods}

\section{Dataset}

The amplification data (see Underlying data $^{12}$ ) obtained with the QIAgen artus HI Virus-1 RT-PCR kit were collected by the Public Health England Clinical Microbiology and Public Health Laboratory, Addenbrooke's Hospital, Hills Road, Cambridge CB2 0QW, UK, during the year 2016. All data were anonymized before use. The reactions were subdivided into clinical samples, control dilutions (CDs), and non-template controls (NTCs). The CDs were based on known dilutions of in vitro transcribed HIV-1 RNA provided by the artus kit, corresponding to $405,4,050,40,500$, and $405,000 \mathrm{c} / \mathrm{mL}$.

\section{Data analysis}

The FP method generated the $\mathrm{Cq}$ by registering the fractional cycle where the fluorescence passed the threshold of 0.2 units. The maxRatio transform of the amplification data and determination of the cut-offs were computed as previously described ${ }^{9,10}$. Different operators visually inspected the reaction's profiles and classified each reaction as either passed or failed. Using R v.3.6, linear models (standard curves, SC) were built on the CDs and applied to calculate the copy numbers according to the formula $10^{(x-b) / m}$ where $x$ is the quantitative cycle (either Cq or FCNA), $b$ and $m$ are the intercept and slope, respectively, of the linear models ${ }^{13}$. VL correlation was obtained with the Pearson product-moment coefficient $\rho^{14}$ and agreement between methods was tested with the Cohen's $\kappa^{15}$; both are reported with their $95 \%$ confidence interval $(\mathrm{CI})$.

\section{Results}

The present dataset was derived from 122 individual artus HIV-1 runs, corresponding to 2,544 reactions (480 CDs, 122 NTCs and 1,931 clinical samples). The cut-offs obtained by EM analysis were multiplied by 2.7 to generate the values used to filter the maxRatio data, as depicted in Figure 1.

The CDs were used to build SCs (Figure 2) that quantified both the CDs (Table 1 and Figure 3) and the clinical samples (Figure 4). Overall, the VLs obtained with the two methods were very strongly correlated $(\rho=0.994,95 \%$ CI: $0.993-0.994)$ and the agreement in the stratification of the reactions into reactive and non-reactive was almost perfect $(\kappa=0.885,95 \% \mathrm{CI}$ : 0.863-0.907). Both methods identified 307 (15.9\%) and 28 $(1.5 \%)$ samples within and above the quantification range 405-405,000 c/mL ( $\rho=0.988,95 \%$ CI: 0.985-0.991 and $\rho=0.992$, 95\% CI: $0.982-0.996$, respectively), and 1,571 (81.3\%) below this range ( $\rho=0.844,95 \%$ CI: $0.829-0.858)$.

FP identified 22 reactions above the limit of detection of $20 \mathrm{c} / \mathrm{mL}$, while maxRatio failed the quantification; conversely, maxRatio identified 18 reactions below $20 \mathrm{c} / \mathrm{mL}$ while FP quantified them above this level. Visual inspection of the amplification profiles of the reactions failed by FP showed that 10 of them (43.4\%) had a proper sigmoid outlook for the internal control (IC) signal that, however, was discarded by the FP (as exemplified in Figure. 5A). In contrast, the others had a low signal for either HIV-1 or IC recovered by maxRatio (Figure 5B). Conversely, $15(83.3 \%)$ of the reactions failed by maxRatio showed either a low IC or HIV-1 input (Figure 6A), whereas the FCNA of the remainder of the reactions produced fractional VL that were rounded to $0 \mathrm{c} / \mathrm{mL}$ (Figure $6 \mathrm{~B}$ ).

\section{Discussion}

The purpose of the present work was to assess the potential benefits of maxRatio in determining HIV-1 VL given its inherent compensation of PCR inhibition. Contrary to our expectation, the SCs we built with either FP or maxRatio were virtually the same. Both methods gave SD VLs significantly divergent from the expected copy numbers at the lower and upper CDs, and maxRatio was, in general, more discrepant from the expected copy numbers than FP. Even concerning the samples' quantification, the two methods produced essentially the same VLs. 

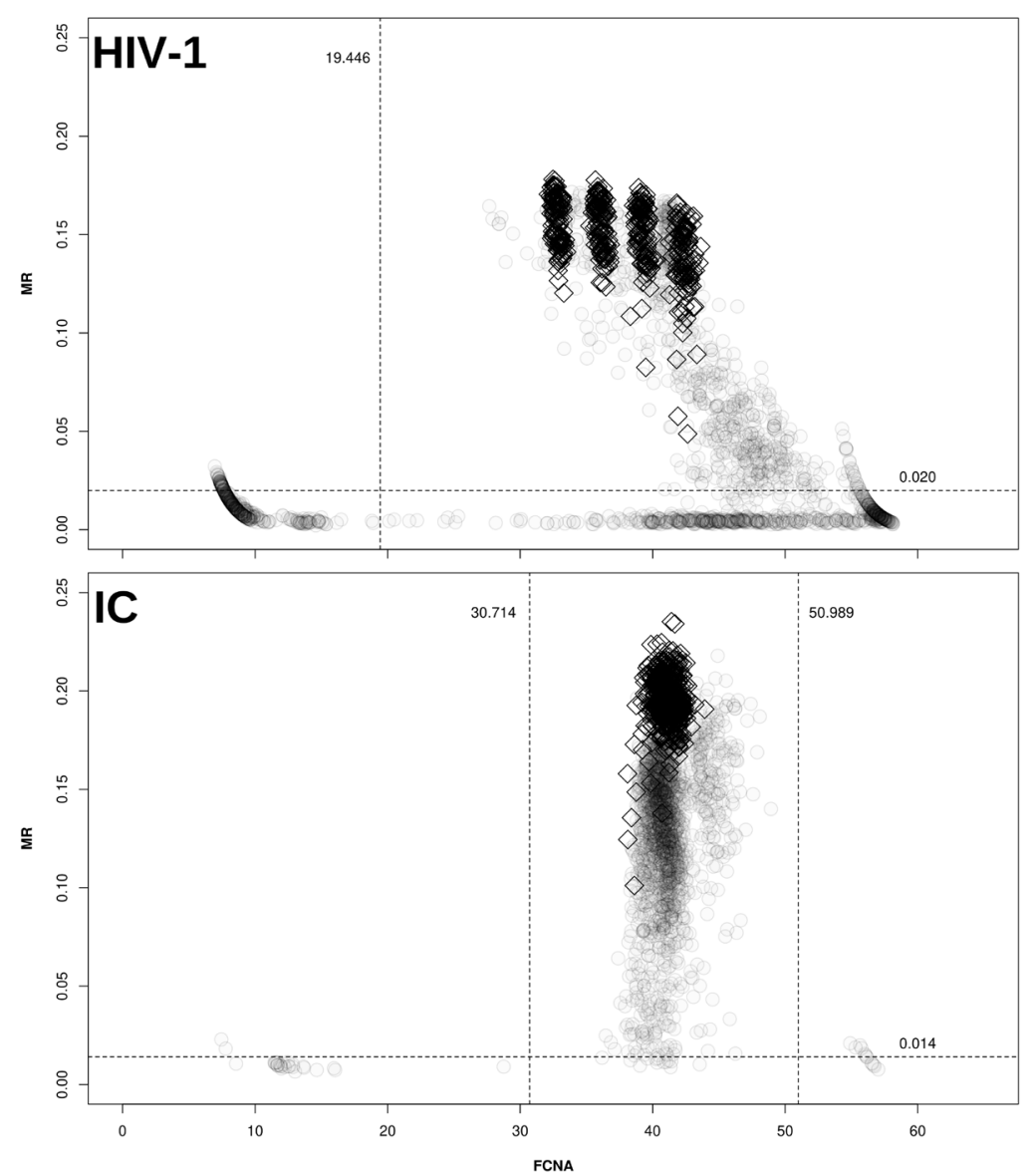

Figure 1. Cut-offs for maxRatio. Clinical samples (o) and CDs $(\diamond)$ are plotted on the maxRatio plane; the numbers report the obtained cut-offs for MR (horizontal lines) and FCNA (vertical lines). A. MR/FCNA pairs for the HIV-1 target. To note how the CDs form four distinct clusters, corresponding to the different standard dilutions. B. MR/FCNA pairs for the IC target. To note that the data form a single cloud because the IC input was virtually the same for all reactions. The presence of two outlier groups at low and high FCNA values required to instantiate two cut-offs.

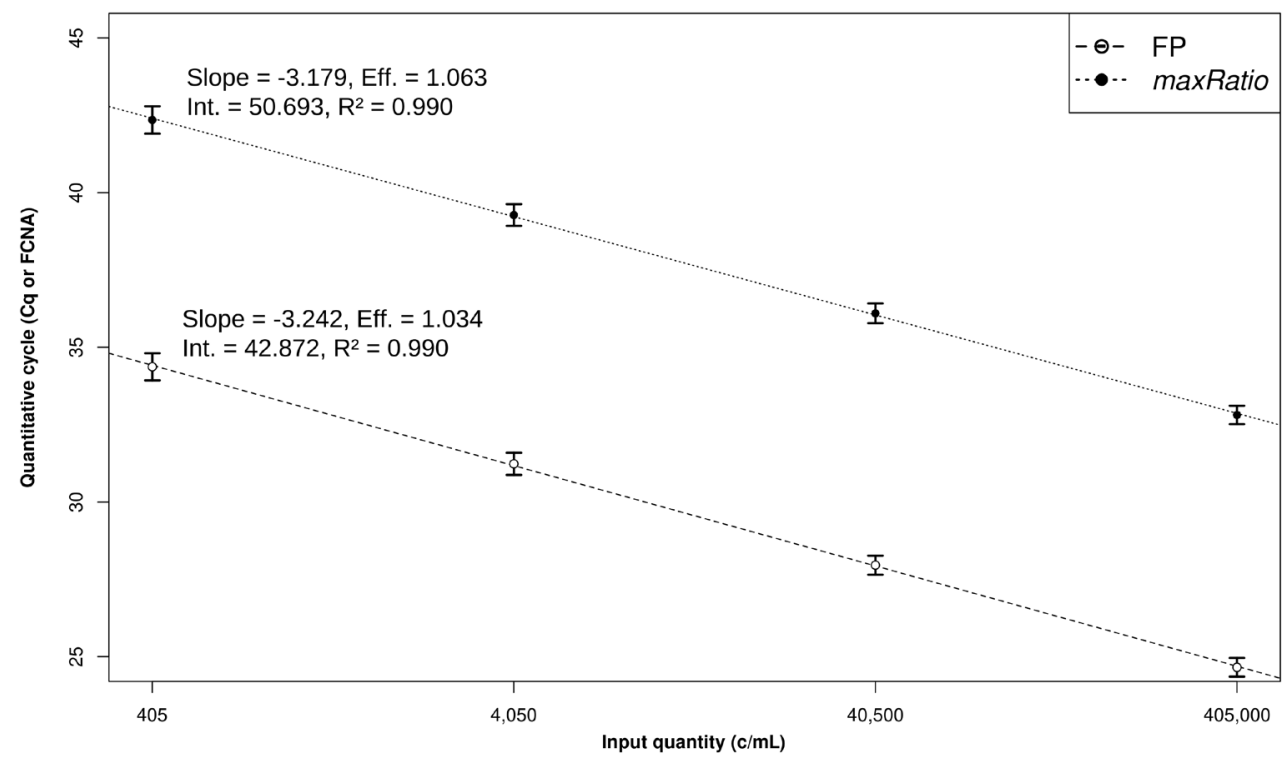

Figure 2. Linear models and CD quantification. Development of the linear models. The Cq (o) and FCNA (•) were used to build SCS for FP (dashed line) and maxRatio (dotted line). The dots and bars represent the mean and standard deviation of the data, respectively. The characteristics of the models are reported. 
Table 1. Comparison of copy numbers for the control dilutions. The mean VL is reported together with the $95 \%$ CI (calculated), the difference between the calculated and the expected concentration (difference), and the result of the t-test (p-value). Statistical significance is represented by * and ** for values below 0.05 and 0.01 , respectively. The copy numbers are given in $\mathrm{c} / \mathrm{mL}$.

\begin{tabular}{|l|c|c|c|c|c|c|}
\hline Dilution & \multicolumn{2}{|c}{ FP } & \multicolumn{4}{c|}{ maxRatio } \\
\hline Expected & Calculated & Difference & p-value & Calculated & Difference & p-value \\
\hline 405 & $440(415-466)$ & 35 & $0.006^{* *}$ & $443(417-469)$ & 38 & $0.004^{\star *}$ \\
\hline 4,050 & $4,014(3,835-4,194)$ & 36 & 0.694 & $4,028(3,843-4,212)$ & 22 & 0.811 \\
\hline 40,500 & $40,777(39,164-42,389)$ & 277 & 0.735 & $40,039(38,385-41,693)$ & -461 & 0.582 \\
\hline 405,000 & $425,384(408,765-442,002)$ & 20,383 & $0.017^{*}$ & $430,673(414,132-447,215)$ & 25,673 & $0.003^{\star *}$ \\
\hline
\end{tabular}
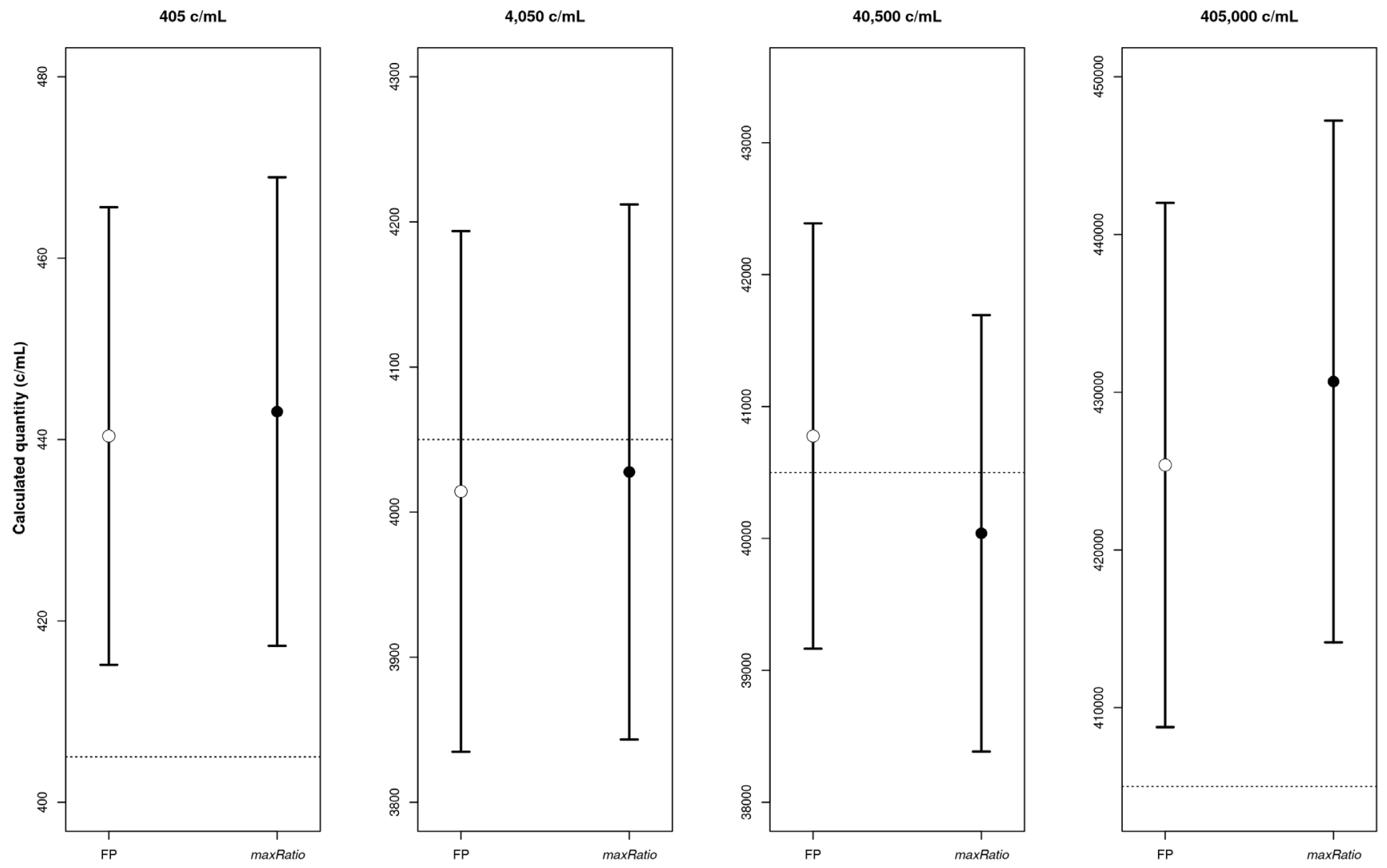

Figure 3. CD quantification. The four panels represent the calculated copy numbers obtained using FP (o) or maxRatio (•) for each dilution. The dots and bars represent the mean and the 95\% CI of the data, respectively.

The main difference between the two methods was in terms of sample's reactivity. By accepting the reactions identified as non-reactive by FP, but reactive by maxRatio, there would have been 18 false-negative results. Conversely, 15 samples identified 


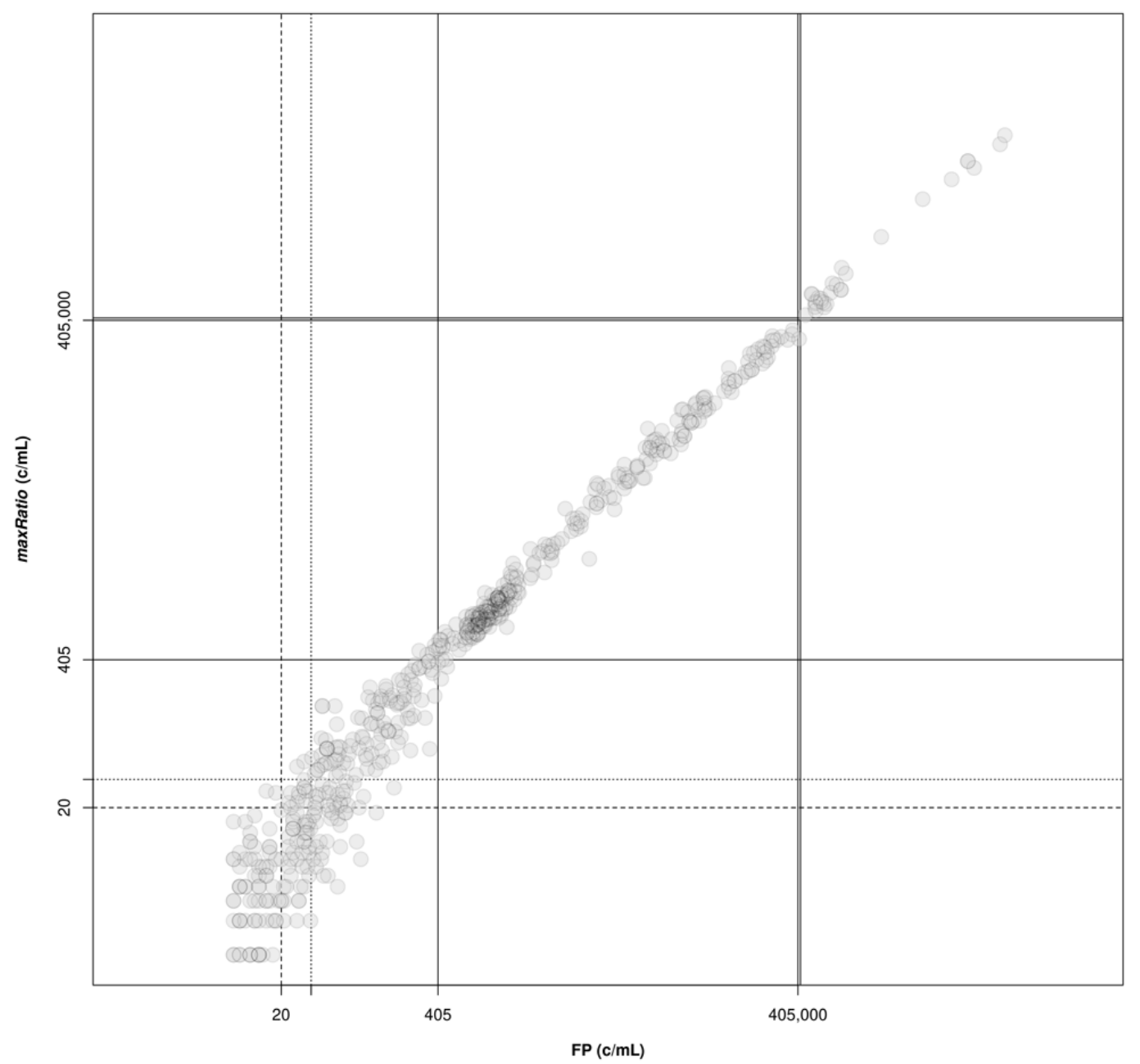

Figure 4. Correlation of VL obtained by FP and maxRatio. The external standard curves were used to calculate the VL for the clinical samples. The following thresholds are depicted: lower (solid lines) and upper (double solid lines) limits of the quantification range; limit of detection of HIV-1 diagnostic in general (dashed line) and artus HI Virus-1 assay in particular (dotted line).

as non-reactive by maxRatio showed aberrant IC that raised quality control, rather than false-positive, issues overlooked by FP.

The current use of maxRatio is to confirm the reactivity determined by FP on the Abbott m2000rt platform. Our data supported this combination as the most effective approach for screening purposes. Samples in disagreement between FP and maxRatio would require further assessment that could reduce the workload involved in issuing the results and minimize the risk of providing false results.
The present work had some limitations. Firstly, the sample size was small. Secondly, the CDs were prepared by diluting the control samples provided in the kit, but the actual concentration was not measured. Finally, we did not have access to the actual issued results; thus, we could not confirm the official VL values.

In conclusion, we compared FP and maxRatio in providing HIV-1 VL. Contrary to our expectations, maxRatio did not give a better quantification than FP, but combining the two methods could minimize issuing false results. 
Sample 374 (Fit point method) $\mathrm{c} / \mathbf{m L}=\mathbf{0}$

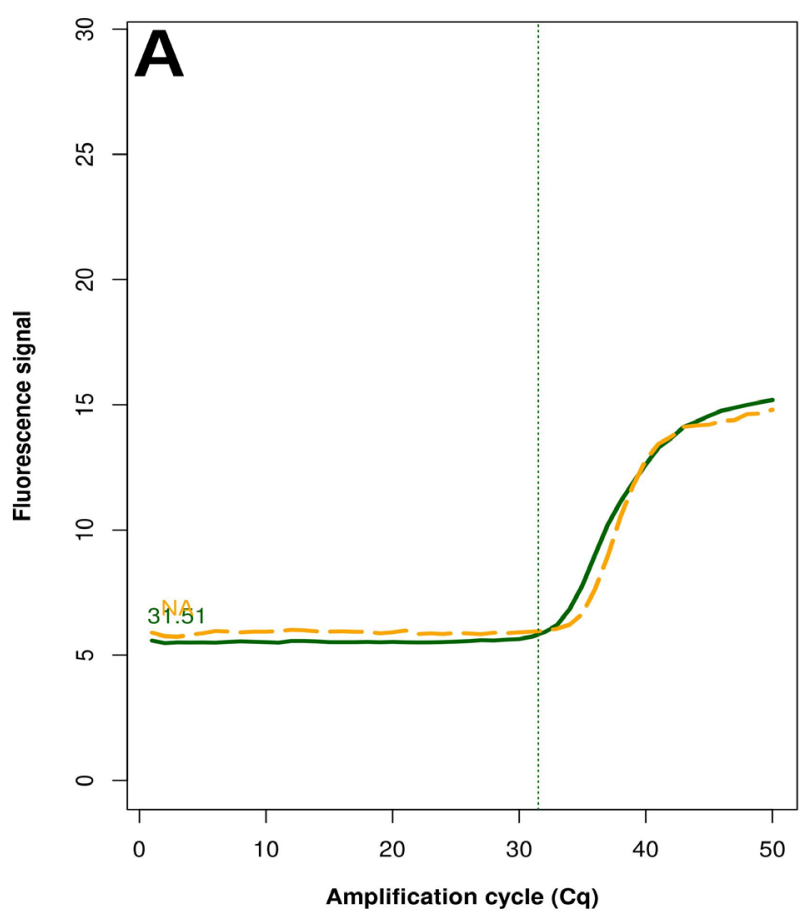

Sample 1256 (Fit point method) $\mathrm{c} / \mathrm{mL}=\mathbf{0}$

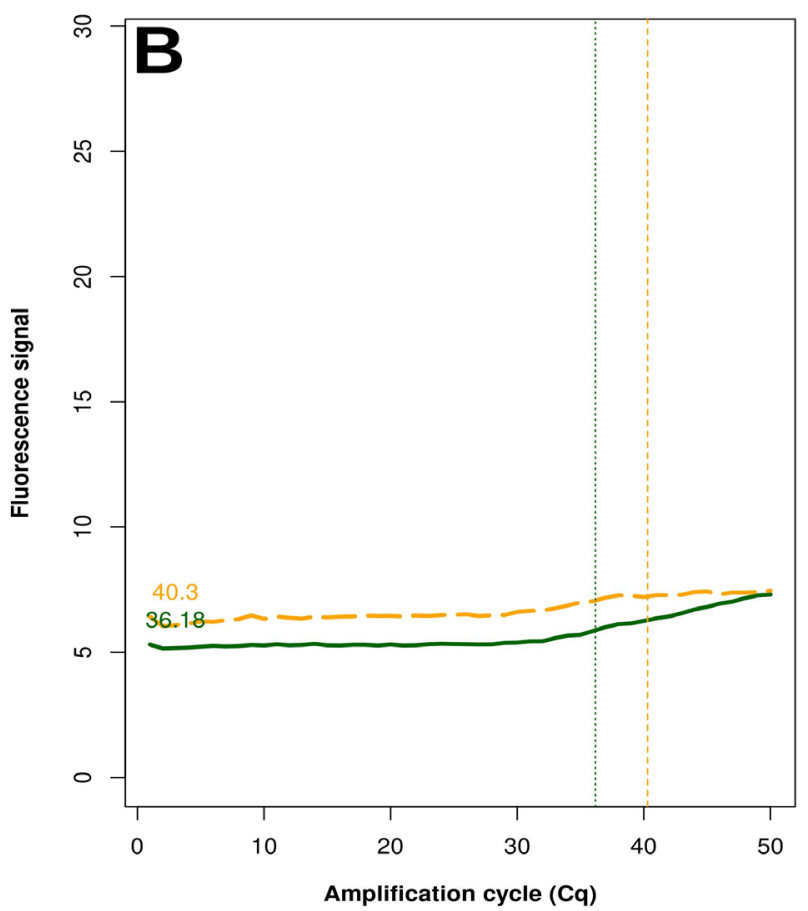

Sample 374 (maxRatio method) $\mathrm{c} / \mathrm{mL}=4693$

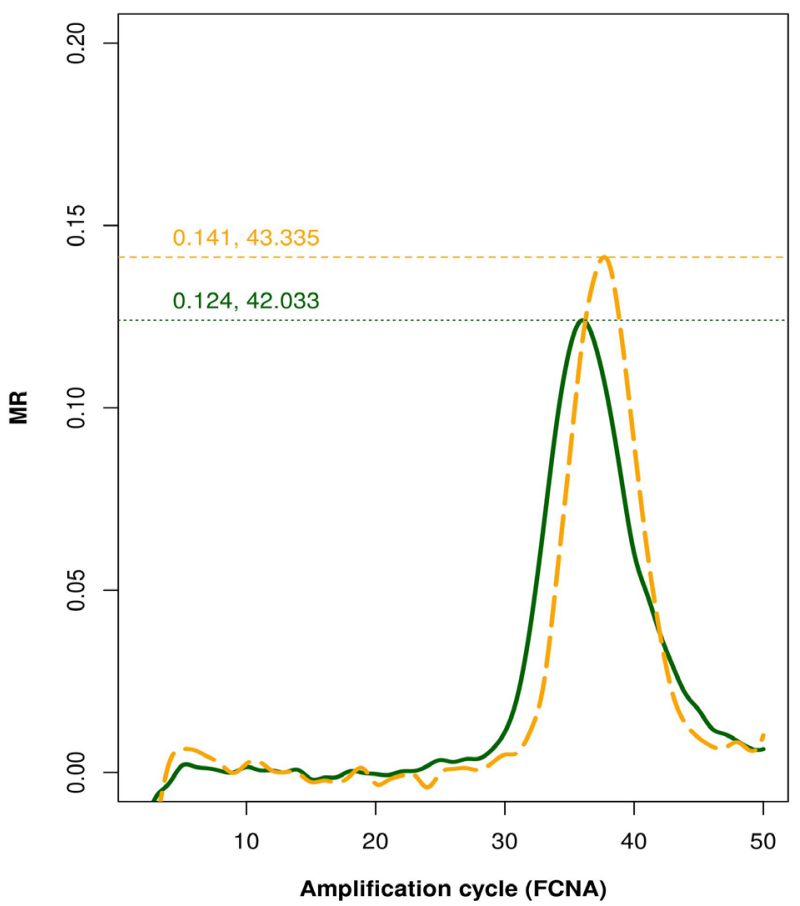

Sample 1256 (maxRatio method) $\mathrm{c} / \mathrm{mL}=1184$

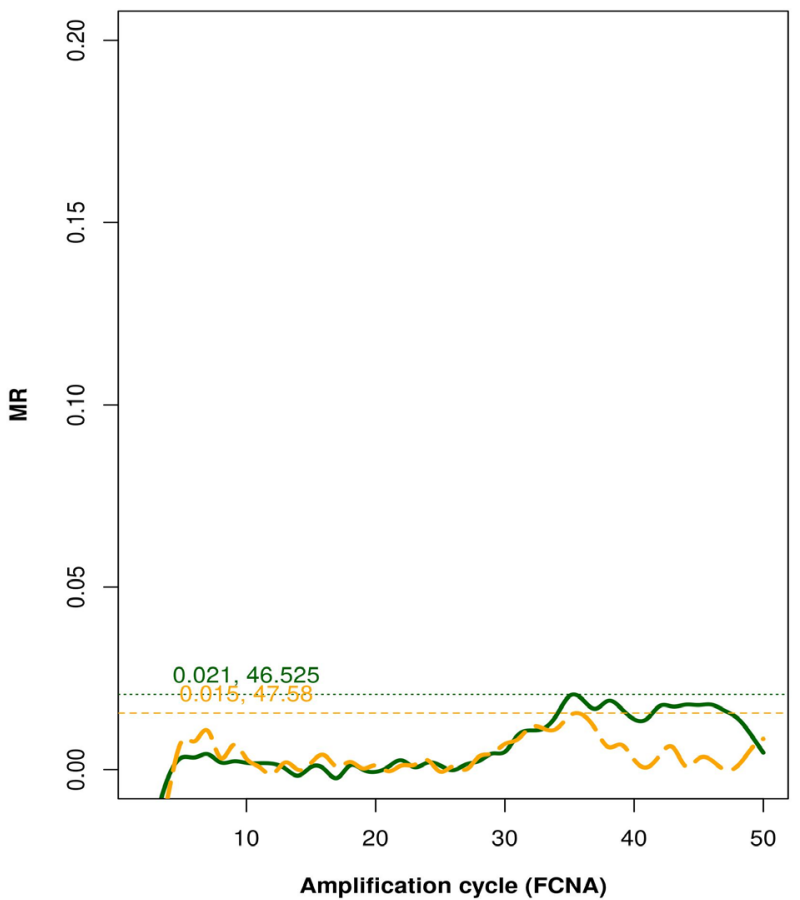

Figure 5. Samples not quantified only by FP. Example of raw amplification profiles for the samples whose FP did not provide a VL. The panels displays the FP (left) the maxRatio (right) transforms of the amplification data. The solid line represents the HIV-1 template and the dashed line the IC template. (A) The majority of the samples had a proper IC profile but the output was undetermined. (B) The minority of the samples showed low target inputs. 

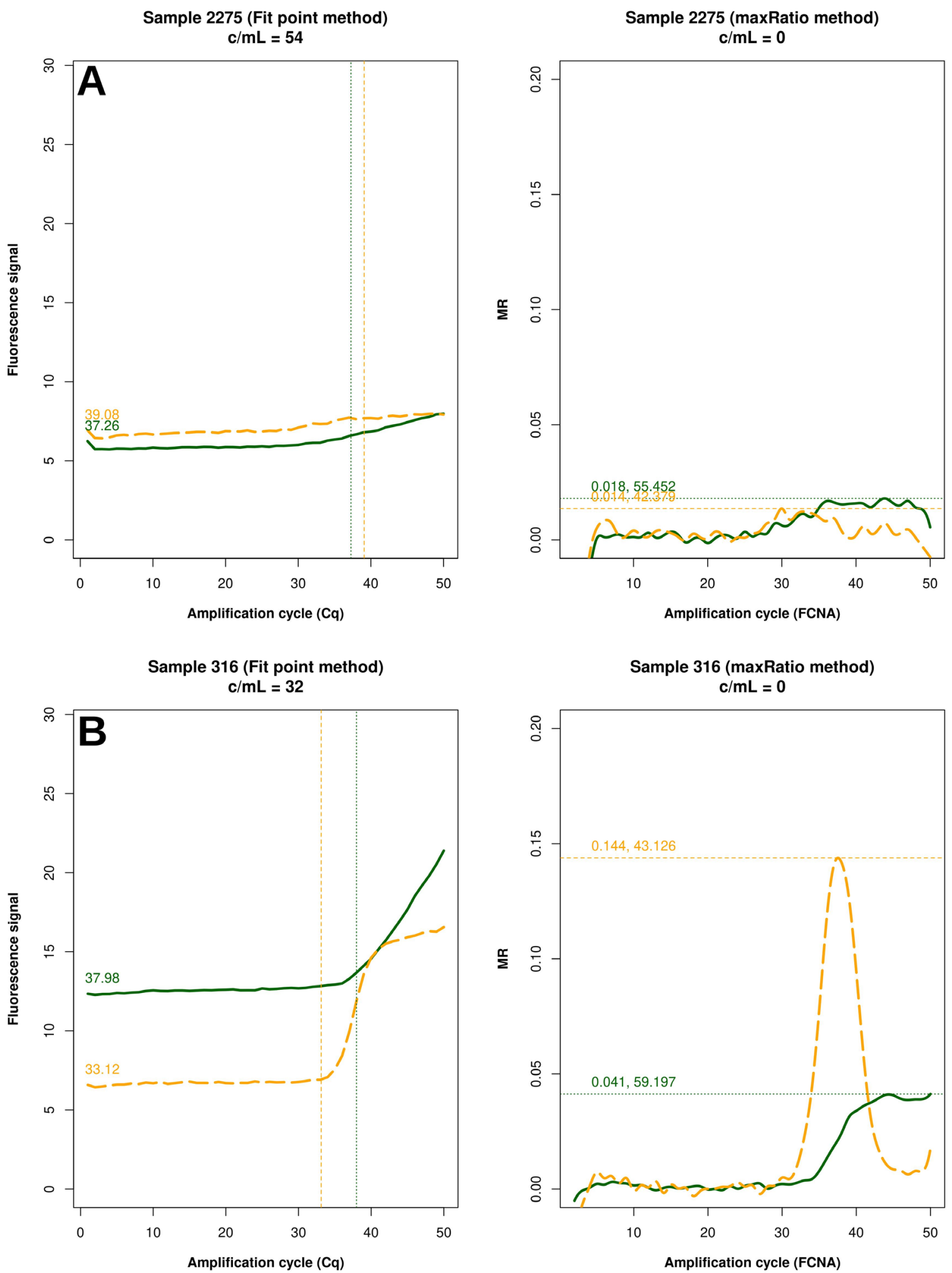

Figure 6. Samples not quantified only by maxRatio. Example of raw amplification profiles for the samples whose maxRatio did not provide a VL. The panels displays the FP (left) the maxRatio (right) transforms of the amplification data. The solid line represents the HIV-1 template and the dashed line the IC template. (A) The majority of the samples had abnormal IC profiles that were overlooked by FP. (B) The minority of the samples showed proper amplification profiles, but the calculation provided fractional VLs that were rounded to zero. 


\section{Data availability}

Underlying data

Harvard Dataverse: artusHIV_amplificationData. https://doi.org/ 10.7910/DVN/0QQNPF

This project contains the following underlying data:

- amplificationDataRaw.tab. (Raw amplification data.)

- viralLoads.tab. (Raw viral loads data.)

Data are available under the terms of the Creative Commons Zero "No rights reserved" data waiver (CCO 1.0 Public domain dedication).

\section{Acknowledgements}

We would like to thank Dr. Martin D. Curran (Public Health England, Clinical Microbiology and Public Health Laboratory, Addenbrooke's Hospital, Cambridge UK) for providing the amplification data.

We would also like to thank Prof. Clementina Elvezia Cocuzza (University of Milano Bicocca, Department of Medicine and Surgery, Monza, Italy) and Dr. Maria Serena Beato ('Istituto Zooprofilattico Sperimentale delle Venezie', Department of Diagnostics in Animal Health, Legnaro, Italy) for their moral support.
1. Wang H, Wolock TM, Carter A, et al.: Estimates of global, regional, and national incidence, prevalence, and mortality of HIV 1980-2015: the Global Burden of Disease Study 2015. Lancet HIV. 2016; 3(8): e361-87. PubMed Abstract | Publisher Full Text | Free Full Text

2. Marschner IC, Collier AC, Coombs RW, et al.: Use of Changes in Plasma Levels of Human Immunodeficiency Virus Type 1 RNA to Assess the Clinical Benefit of Antiretroviral Therapy. J Infect Dis. 1998; 177(1): 40-7. PubMed Abstract | Publisher Full Text

3. Adams P, Vancutsem E, Nicolaizeau C, et al.: Multicenter evaluation of the cobas $₫$ HIV-1 quantitative nucleic acid test for use on the cobas ${ }^{\circledR} 4800$ system for the quantification of HIV-1 plasma viral load. J Clin Virol. 2019; 114: $43-9$. PubMed Abstract | Publisher Full Text

4. Sizmann D, Glaubitz J, Simon CO, et al.: Improved HIV-1 RNA quantitation by COBAS $₫$ AmpliPrep/COBAS $®$ TaqMan $₫$ HIV-1 Test, v2.0 using a novel dualtarget approach. J Clin Virol. 2010; 49(1): 41-6. PubMed Abstract | Publisher Full Text

5. Bustin SA: Absolute quantification of mRNA using real-time reverse transcription polymerase chain reaction assays. J Mol Endocrinol. 2000; 25(2): 169-93.

PubMed Abstract | Publisher Full Text

6. Bar T, Kubista M, Tichopad A: Validation of kinetics similarity in qPCR. Nucleic Acids Res. 2012; 40(4): 1395-406.

PubMed Abstract | Publisher Full Text | Free Full Text

7. Chen $P$, Huang X: Comparison of Analytic Methods for Quantitative RealTime Polymerase Chain Reaction Data. J Comput Biol. 2015; 22(11): 988-96.

PubMed Abstract | Publisher Full Text | Free Full Text
8. Ramakers C, Ruijter JM, Lekanne Deprez RH, et al.: Assumption-free analysis of quantitative real-time polymerase chain reaction (PCR) data. Neurosci Lett. 2003; 339(1): 62-6.

PubMed Abstract | Publisher Full Text

9. Shain $\mathrm{EB}$, Clemens JM: A new method for robust quantitative and qualitative analysis of real-time PCR. Nucleic Acids Res. 2008; 36(14): e91. PubMed Abstract | Publisher Full Text | Free Full Text

10. Marongiu L, Shain E, Drumright L, et al.: Analysis of TaqMan Array Cards Data by an Assumption-Free Improvement of the maxRatio Algorithm Is More Accurate than the Cycle-Threshold Method. PLoS One. 2016; 11(11): e0165282.

PubMed Abstract | Publisher Full Text | Free Full Text

11. Marongiu L, Shain $\mathrm{E}$, Shain $\mathrm{K}$, et al.: Filtering maxRatio results with machine learning models increases quantitative PCR accuracy over the fit point method. J Microbiol Methods. 2020; 169(1): 105803. PubMed Abstract | Publisher Full Text

12. Marongiu L: "artusHIV_amplificationData”. Harvard Dataverse, V1, 2020. http://www.doi.org/10.7910/DVN/OQQNPF

13. Scott Adams P: Data Analysis and Reporting. In: Dorak T editor. Real-time PCR. 1st ed. New: Taylor \& Francis; 2006; 39-62. Reference Source

14. Schober P, Schwarte LA: Correlation coefficients: Appropriate use and interpretation. Anesth Analg. 2018; 126(5): 1763-8. PubMed Abstract | Publisher Full Text

15. Viera AJ, Garrett JM: Understanding interobserver agreement: The kappa statistic. Fam Med. 2005; 37(5): 360-3.

PubMed Abstract 


\section{Open Peer Review}

\section{Current Peer Review Status:}

\section{Version 1}

Reviewer Report 10 June 2021

https://doi.org/10.5256/f1000research.28406.r83772

(C) 2021 Panieri E. This is an open access peer review report distributed under the terms of the Creative Commons Attribution License, which permits unrestricted use, distribution, and reproduction in any medium, provided the original work is properly cited.

\section{Emiliano Panieri}

Department of Physiology and Pharmacology, Faculty of Pharmacy and Medicine, Sapienza University of Rome, Rome, Italy

\section{General comment:}

The paper from Marongiu et al. presents an alternative approach to the conventional use of fitpoint (FP) or MaxRatio methods, which can be used to accurately determine the viral load of HIV-1 patients under experimental conditions wherein the amplification efficiency might vary across the samples, as in the case of low-copy number of viral RNA or in presence of PCR inhibitors. This is a clinically relevant issue since the VLs widely differ among HIV-1 infected individuals, potentially leading to issues of false-positive/negative results when the viremia is very low or the efficiency of $P C R$ reactions is not uniform. It is therefore of utmost importance to improve not only the analytical sensitivity of qPCR methods but also their predictive value to avoid that a substantial fraction of HIV-1 patients will be incorrectly diagnosed, causing a negative impact on their prognosis and treatment monitoring. In this regard, the approach proposed by the authors, based on both FP and MaxRatio is straightforward and when correctly applied can represent a costeffective alternative to the time-consuming and labor-intensive work required to validate false positive and false negative results. In general, the manuscript is well written, concise and presented in a clear, scientifically-sound way. The data are quite exhaustive, corroborated by appropriate statistical analysis and firmly support the authors' conclusions.

However, some minor changes will be required to improve the MS quality, which are listed below: The authors correctly indicate that the small-size of the tested samples represents a limitation to their study. Can also the authors discuss whether increasing the sample size is expected to proportionally increase the accuracy of the proposed method? In other words, in the authors' opinion, is the number of tested samples expected to influence the predictive value of the FP + MaxRatio use? It might be useful to further elaborate this aspect in the discussion to help the readers to gain insights on this limitation.

The authors should indicate which kind of t-test was used to assess the statistical significance of the data contained in Table 1 (i.e paired or unpaired, with or without other 
corrections such as Welch's or Bonferroni's) and which software was used to perform the statistical analysis.

In the Results paragraph it is suggested to mitigate the following statement: "...the agreement in the stratification of the reactions into reactive and non-reactive was almost perfect ( $\mathrm{k}=0.885,95 \% \mathrm{CI}: 0.863-0.907)$. ". Instead, "noticeably robust" can be a good alternative.

Few lines below it is suggested to slightly rearrange the sentence "...the FP identified 22 reactions above the limit of detection of $20 \mathrm{c} / \mathrm{mL}$, while maxRatio failed the quantification" (i.e. while Max Ratio was unsuccessful).

Few lines below it is suggested to substitute "sigmoid outlook" with "sigmoidal shape".

Few lines below it is suggested to change "...of the remainder of the reactions" into "...of the remaining reactions".

In the Figure 1 legend, the authors describe a panel A (MR/FCNA pairs for the HIV-1 target) and a panel B (MR/FCNA pairs for the IC) which are however absent in the Figure 1. Please add the correct indication or either refer to the panels as upper and lower.

In the Figure 5-6 legend, it should be corrected "the panels displays" with "the panels display".

In the last three lines of the Discussion section please rephrase the sentence: "...maxRatio did not give a better quantification than FP, but combining the two methods could minimize issuing false results" into "maxRatio did not result in a better quantification than FP but when combined together these two methods could..."

Is the work clearly and accurately presented and does it cite the current literature? Yes

Is the study design appropriate and is the work technically sound?

Yes

Are sufficient details of methods and analysis provided to allow replication by others? Yes

If applicable, is the statistical analysis and its interpretation appropriate? Yes

Are all the source data underlying the results available to ensure full reproducibility? Yes

Are the conclusions drawn adequately supported by the results? Yes 
Competing Interests: No competing interests were disclosed.

Reviewer Expertise: Oncology, cellular and molecular biology

I confirm that I have read this submission and believe that I have an appropriate level of expertise to confirm that it is of an acceptable scientific standard.

The benefits of publishing with F1000Research:

- Your article is published within days, with no editorial bias

- You can publish traditional articles, null/negative results, case reports, data notes and more

- The peer review process is transparent and collaborative

- Your article is indexed in PubMed after passing peer review

- Dedicated customer support at every stage

For pre-submission enquiries, contact research@f1000.com 